\title{
Progress on the development of a simulation environment for optical communications (Withdrawal Notice)
}

Joel Shields, Meera Srinivasan, Martin Regehr

Joel Shields, Meera Srinivasan, Martin W. Regehr, "Progress on the development of a simulation environment for optical communications (Withdrawal Notice)," Proc. SPIE 8246, Free-Space Laser Communication Technologies XXIV, 82460X (22 February 2012); doi: 10.1117/12.913567 


\section{Progress on the development of a simulation environment for optical communications (Withdrawal Notice)}

Proc. SPIE 8246, 82460X (2012); http://dx.doi.org/10.1117/12.892581

Online Publication Date: 22 February 2012

Withdrawn from Publication: 2 March 2012

Conference Date: Tuesday 24 January 2012

Conference Location: San Francisco, California, USA

Conference Title: Free-Space Laser Communication Technologies XXIV

Conference Chairs: Hamid Hemmati, Don M. Boroson

Joel Shields, Meera Srinivasan, and Martin W. Regehr

Jet Propulsion Lab. (United States)

This paper was presented at the SPIE conference indicated above and has been withdrawn from publication at the request of the authors. 